\title{
L'éducation médicale vue par un philosophe
}

\author{
Medical education through the eyes of a philosopher
}

\author{
Michel SERRES*
}

\begin{abstract}
Résumé Propos: Les médecins doivent développer et entretenir parallèlement deux têtes : l'une, scientifique, l'autre empirique. Cette exigence résulte de la nécessité de solliciter à la fois la raison et l'expérience, d'appréhender tout autant le général et le particulier, la notion stable et la mouvance de la singularité, la maladie et la personne souffrante. Exégèse : Jadis, la maladie passait pour normale plus que pour fatale ou pénible, alors que la santé passe aujourd'hui pour usuelle; la normalité a changé de camp. Il est licite de sinterroger quant à savoir si l'on peut définir la vie humaine de la même façon lorsquelle dure vingt ou quatre-vingts ans. Parallèlement, la science doit composer avec les puissances de l'argent, des médias et de l'administration. Le siècle dernier a suscité l'avènement d'une bioculture, faite de sciences et de pratiques du vivant, tous règnes confondus. La vie, dans sa totalité, entre dans l'action et la responsabilité de l'homme. L'avenir de la médecine se joue à ce niveau évolutif. Les variations sur le thème médecin, à partir de la racine linguistique "mode", conduisent à rapprocher: médication, méditation, mesure, modéré, modeste. Conclusions : La pratique et l'éducation médicales doivent sinscrire entre les tensions que constituent la nécessité d'une santé, la possibilité des existences, les impossibilités induites par la maladie et la douleur et la contingence de l'action du médecin. Pour atteindre le singulier, l'apprentissage de la médecine doit s'appuyer aussi sur la culture, telle qu'y contribuent par exemple les grands écrivains et que l'on retrouve dans les textes de portée universelle, car ces auteurs explorent et décrivent des expériences individuelles telles que les rencontrera le médecin et qu'assurément il manquera si, limité à la raison brute, il reste un instruit inculte.
\end{abstract}

Abstract Purpose: Doctors have to develop and to maintain in parallel two heads: one scientific and the other empiric. This requirement results from the need to solicit at the same time reason and experience, to apprehend the overall just as well as the distinctive, the stability and the ever changing of singularity, illness and pain. Exegesis: In the olden days, illness was considered normal more than fatal or painful, whereas today, health is mostly common: normality has changed sides. It is licit to wonder if we can define a life of 20 years old identical as a life of 80 years old. At the same time, science has to deal with the power of money, media and management. The last century has aroused the advent of bioculture, made up of both science and life practice. Life, in its entirety, depends on mankind actions and responsibilities. The future of medicine will be tributary of this evolutionary level. Variations of the theme medicine from the linguistic root "mode" lead to relate: medication, meditation, measure, moderate, modest. Conclusions: medical practice and medical education have to emerge from tensions involving health requirement, the possibility of life, impossibilities induced by illness and pain and finally, the contingency of an action from a doctor. To reach the singular, medicine learning should also rely on culture, which comprises written work from great authors universally recognized: these authors have explored and described individual experiences in which the physician can relate to and, if the latter is only limited to its reason, he will remain an uneducated medical expert.

Pédagogie Médicale 2006;7:135-41

1- Texte de la conférence prononcée en ouverture du $1^{\text {er }}$ Congrès de la Société internationale francophone d'éducation médicale (SIFEM), Beyrouth 1, 2 juin 2006

* Membre de l'Académie Française 


\section{Portrait}

Amis médecins, vos têtes me fascinent. Elles ne ressemblent à nulle autre. J'ai longtemps cru que vous n'en aviez qu'une, comme tout le monde. Or, à y bien regarder, je vois que vous en portez deux au bout du cou. L'obligation où vous met votre pratique de tenir un cap difficile entre les formes canoniques d'une maladie et le corps toujours inattendu de cette patiente ou de ce malade, entre l'apprentissage abstrait de votre jeunesse et une expérience humaine dont le profil ne cessant de fluctuer vous amène à douter de cet enseignement pourtant toujours là ; bref, les vibrations subtiles entre l'idée générale et la personne concrète, la notion stable et la mouvance de la singularité irréductible... font de vous des sages qui savent que l'individu résiste en substance aux idéalités pourtant nécessaires à sa compréhension et que nous ne pouvons saisir la vie sans ce double apport contradictoire. D'où votre bizarre bicéphalie entée sur tige bifide : quelques-uns, parmi vous, les jeunes surtout, montrent un gros crâne scientifique et un petit, empirique, alors que les plus âgés ont tendance à inverser la proportion, car la sollicitude, chez eux, les a souvent rendus sceptiques au sujet des raisons, pendant que s'épanouissait leur cœur.

Portant sur les épaules savoir et pitié, le Tiers-Instruit, c'est vous. À deux cerveaux, à langue double, à deux paires d'yeux et d'oreilles, cette bicéphalie cultivée vous distingue de nous, vos patients, comme notre chance et votre expertise. Nul ne pense comme vous, nul ne sent ni n'agit comme vous, mon chef unique s'en trouve jaloux. Exemplaires, vos deux têtes dont vous veillez tous les jours à la croissance parallèle, résument, à mon sens, les conduites humaines, lorsqu'elles excellent dans l'intelligence ; l'une reste dans la science, l'autre plonge dans le paysage. Une bouche dit diabète, l'autre cite le prénom de celle qui en souffre. En ce tête-à-tête permanent gît le secret transhistorique de la médecine.

\section{Histoire}

Or, en plusieurs millénaires, la balance entre ces deux têtes changea, par équilibres rares et déséquilibres fréquents : deux chefs font rarement bon ménage. Vous connûtes donc des doctes aussi formalistes que Galien, loin des réalités tangibles, et d'autres praticiens plus hippocratiques, consacrés aux humeurs des individus ou attentifs, comme Vésale, à disséquer des cadavres. Ainsi votre histoire peut se résumer à grands traits par ce dialogue virant parfois à la chamaille et qui produisit une évolution en perpétuelle bascule où la victoire court parfois vers la raison et quelquefois vers l'expérience. Or encore, cette aventure à l'aveugle et, il faut l'avouer, marquée de fréquentes défaites, accéda, voici un demi-siècle, à des triomphes indiscutables, obtenus par une des deux têtes, la ratiocinante et lucide. Dernière bascule, décisive celle-là, sans doute définitive, fondatrice en tous cas de la nouvelle médecine.

Depuis Pasteur, pur cristallographe, en effet, depuis Schrödinger, physicien atomiste, suivis d'un cortège de biochimistes en foule, Watson, Pauling, Monod... experts en macro-molécules, vous perdîtes cette tête, ces laborantins vous la volèrent ; depuis un demi-siècle, vous fûtes même dépossédés par eux du prix Nobel de médecine. En explorant l'invisible, ils découvrirent mille secrets pour produire autant de remèdes. La rationalisation et l'objectivité qui s'ensuivit aboutirent, pour la première fois dans l'histoire, à des guérisons innombrables. Or, si l'art médical remonte à la nuit des temps et remplit l'universalité des cultures humaines, la guérison courante, quant à elle, date à peine de quelques décennies et du monde occidental. Jusqu'aux années 1950, en effet, les médecins soignaient, certes, diagnostiquaient, pronostiquaient... souvent même avec finesse et précision, mais disposaient de si peu de moyens pour vaincre la maladie que le plus souvent ils devaient s'en remettre, pour cela, au Dieu de leur foi, à la nature, à l'aléa. Comme disent les médecins de Molière, nous avons changé tout cela. Un immense événement a déchiré l'histoire : le praticien soignait toujours, il guérit désormais souvent. Cette nouveauté, nous en mesurons encore mal les conséquences évolutives, psychologiques, sociales, politiques et anthropologiques.

Car intervint, d'autre part, et aussi pour la première fois, une deuxième objectivité touchant le collectif, sous trois formes. L'une, scientifique à nouveau, énonça des statistiques et l'on put traiter, maladies et santé, sur de grandes populations ; la deuxième, en conséquence, décida de politiques de prévention ; la troisième, solidaire, organisa la gratuité des soins, au moins relative et variable selon les pays. À l'événement historique de la guérison s'ajoutèrent donc des stratégies d'ensemble dont nous savons aujourd'hui mesurer l'efficacité. Si guérir changea les corps individuels, l'intervention du groupe changea la société.

Ne voyez, par exemple, nul paradoxe, mais seulement un souvenir dont je témoigne sans mentir, dans cette mienne affirmation aux termes de laquelle, jadis et naguère, la maladie passait pour normale plus que pour fatale ou pénible, alors que la santé passe aujourd'hui pour usuelle. 
Oui, la normalité a changé de camp ; elle se tenait, au moins statistiquement, du côté du pathologique, mes successeurs ont de la peine à l'imaginer. Car lorsque les malades ne guérissaient que rarement, tout le monde luttait, plus ou moins dans la peine, pour se rétablir, et cela, usuel, se voyait peu; nous ne redoutons plus désormais que les risques, parce que ce fut la santé qui devint un état partagé ; cette perception commune qui, à son tour, change le regard médical, transforme les groupes et les hommes. Peut-être n'avons-nous pas encore dépassé ce point pour les maladies de l'âme.

Je ne puis entrer ici dans tous les détails du tableau. Voici, à grands traits. Maladies infectieuses : avant la deuxième guerre mondiale, un cabinet médical recevait plus de syphilitiques et de tuberculeux que d'autres patients. Vingt ans après, il ne restait plus que ces autres. Espérance de vie : demain, disent les démographes, pulluleront les centenaires; dans la préhistoire, $50 \%$ des nouveaux-nés mouraient, la moitié des survivants ne dépassaient pas neuf ans et le reste disparaissait entre dix-huit et vingtcinq. Toute chair passait comme l'herbe et comme la fleur des champs. Déjà rangée chez Balzac, la femme de trente ans brille aujourd'hui de prime jeunesse. Ainsi, la courbe de l'espérance de vie croît lentement, de manière quasi plate, au cours de l'histoire, pour se redresser soudain, immédiatement après l'événement dont je parle. Peut-on définir la vie humaine de la même façon lorsqu'elle dure vingt ou quatre-vingts ans ? Souffrance : protégé par les meilleurs médecins de son royaume, Louis XIV cria de douleur tous les jours ; un praticien contemporain peut recevoir dans son cabinet une ou un septuagénaire, privés de rang ou de fortune, sans expérience expresse de la souffrance. Lirionsnous les belles pages de Montaigne sur la pierre, s'il avait bénéficié de notre pharmacie? Taille et apparence : les soldats de la Grande Armée napoléonienne mesuraient $1,55 \mathrm{~m}$ en moyenne, Victor Hugo les acclamait sous le nom de géants ; disparus pour cause de paix européenne, nos conscrits atteignent $1,78 \mathrm{~m}$; emballées sous crinolines et falbalas pour dissimuler cicatrices et bubons, voici nos belles sur la plage d'août, nues parce que lisses. Ce bref tableau montre deux corps : je ne sache pas qu'ils se ressemblent.

\section{De l'bistoire à l'anthropologie: le corps nouveau}

Nous n'entretenons plus les mêmes rapports à la douleur, à la mort, aux autres vivants et au monde, que nos prédé- cesseurs, même proches, nous ne nous alimentons pas de la même façon, nous ne vivons plus dans le même temps ni en longueur ni en rythme, nous changeons même tantôt de reproduction : lorsque nous faisions des enfants à l'aveuglette, nous suivions le temps contingent de la naissance; dès que nous manipulons le génome, nous ouvrons un éventail énorme de possibles temporels. Que son corps ne naisse ni ne meure de la même façon, ne souffre ni ne se nourrisse des mêmes aliments, ne vive ni ne se développe dans le même espace et dans le même temps, dans un autre monde et une nouvelle société, sáagit-il vraiment du même homme? La mutation historique de la médecine aboutit alors à une évolution, au sens darwinien, du corps humain. J'ai nommé ce processus : hominescence.

Objet de science, notre corps changea donc, au cours du dernier demi-siècle de façon si complète que le praticien ne rencontre plus le même patient et que le collectif change de santé. L'histoire récente des pratiques médicales aboutit donc à un résultat qui dépasse de loin ces moyens de sorte que le temps lui-même bifurque. Autrement dit, alors que je raconte le changement des soins et des remèdes dans celui de l'histoire, cette transformation corporelle plonge dans une durée de type évolutif. Certes, je pourrais écrire vingt récits, passer en revue l'imagerie et la pharmacie, les politiques de vaccination, de prévention et de santé, le travail allégé, l'assainissement des habitats, l'expansion de l'hygiène... reste que ces histoires convergent vers un résultat plus ample que les composantes de ses causes : cette transformation anthropologique des corps. Ce changement bénéficia des moyens découverts par les savoirs et appliqués par les institutions et les hommes de l'art, dont on a écrit vingt fois les annales, mais la fin dépassa lesdits moyens au point que si ceux-ci peuvent se relater dans le temps de l'histoire, celle-là entre, j’insiste, dans l'évolution. Honteux, ce disant, de ne parler que des pays riches, je vis difficilement, comme beaucoup, une inégalité qui leur dénie le titre de démocraties ; nous paierons un jour cette injustice.

En grande partie, la science surtout produisit ce changement local majeur. Qui ne se réjouirait donc de ce que la première tête médicale engrange chimie et biophysique, puisque le principal des réussites thérapeutiques vient aujourd'hui de la recherche fondamentale, des molécules et des techniques de tous ordres? Nous ne pouvons, nous ne devons revenir en arrière en aucune façon. De la découverte des antibiotiques à l'éradication de la petite vérole, de l'échographie au scanner, des analgésiques aux tranquillisants, les laboratoires précèdent et causent souvent la 
guérison. Et nous n'en avons pas fini avec eux : nous commençons à peine d'exploiter les possibilités des nouvelles technologies qui permettent les consultations à distance et des biotechnologies encore dans l'embryon. Aux militants des mouvements anti-science, je recommande donc d'essayer l'ignorance qu'ils paraissent chérir, bien qu'elle échoue avec régularité depuis la fondation du monde.

Mais ce changement dont je ne crois pas exagérer la portée connut, peu à peu, une contre-partie. La rationalisation médicale qui s'ensuivit et dont la science ne porte pas, et de loin, toute la responsabilité, compliqua parfois la vie des patients, mais aussi celle des médecins. Les premiers, devenus quelquefois orphelins de celui qui, de jadis à naguère, les accompagnait personnellement, comme l'archange Raphaël, un autre de vos ancêtres, suivit le jeune voyageur Tobie pas à pas, en lui conseillant des spécifiques inouiis, ne surent bientôt plus qui les soignait par le dédale des hôpitaux, l'illisibilité des analyses et les guichets des organismes publics ; le médecin, redouta de se trouver, quant à lui, peu à peu dessaisi de ce qui faisait de sa pratique un art tout humain : le rapport privé à la personne. Ces malaises surviennent, ô paradoxe, au moment même des plus grandes réussites de la médecine. Impuissante, elle avait bonne presse ; victorieuse, l'opinion en doute. Apparurent même des modes étranges dont l'obscurantisme lutte contre cette science même à qui nous devons le nouveau corps. Crise sans exemple.

\section{Jeu à quatre}

Je précise. Après avoir installé les portraits du médecin et de son patient dans leurs dimensions historique et anthropologique, je reviens un moment aux sociétés modernes qui ont connu ces victoires. Vue de l'extérieur, la science qui soigne et guérit efficacement y compose sa rationalisation avec celle de l'argent qui finance les laboratoires et en tire son profit, celle des médias qui annoncent les catastrophes et celle de l'administration qui ordonne et organise. Ce jeu à quatre, compliqué, dessine assez précisément les mouvements, parfois chaotiques, des collectifs occidentaux où toute accusation, en cas de malheur, finit par viser l'un des ces quatre acteurs, généralement le plus faible : vous.

Je distinguais volontiers autrefois trois pouvoirs dominants : médias, science et administration, parce qu'ils ne rencontrent devant eux aucun contre-pouvoir. Qui s'oppose à la vérité se trompe ou ment ; comment critiquer les canaux principaux de la parole et de l'image sans les emprunter? La loi met en examen celui qui ne suit pas le droit. Qui donc peut s'opposer à des pouvoirs sans balance ? Or, ils projettent et organisent dans la société trois fonctions fondamentales du langage : la science s'efforce d'atteindre sa valeur de vérité, les médias exhibent sa puissance de séduction et l'administration impose sa force performative. Quant à l'argent, sa puissance provient de l'équivalent général, sans besoin de traduction, plus universel encore donc que toute langue.

La philosophie tente de comprendre bien des choses, mais quel profond génie expliquera un jour la mystérieuse croissance somme toute récente, immense, inévitable, rarement justifiée, dont la multiplication cancéreuse vitrifie sur son passage la science elle-même et sa recherche intelligente, l'enseignement et ses transmissions, mais aussi bien l'architecture ou l'urbanisme et leurs aises disparues, le lien social et la politique, tout en somme et en particulier la médecine et sa pitié... le tsunami géant de -ouvrez bien la bouche avant qu'elle vous étouffe, pour mieux prononcer sa première syllabe- l'Administration ? Sans expertise, elle décide de la recherche et de la formation ; sans connaissance du terrain, y commande ; sans expérience, dicte quand, comment et qui soigner... Dossiers, bureaux clos et textes de lois, indéchiffrables, partagent le pouvoir avec les Médias, bavards plats, sans opposition possible. Mais, inversement, comment, sans l'Administration, lutter contre le pouvoir blanc et universel de l'argent ? Le praticien, enfin, s'inquiète de voir la société laisser à une jurisprudence sans expertise le soin de définir, procès après procès, le vivant et ses normes; de quel droit le droit s'arroge-t-il ce nouveau droit de vie et de mort ? Comme au jeu du ciseau, de la feuille et de la pierre, chacune de ces puissances, tour à tour, prend le pouvoir sur les autres pouvoirs, sans changement notable pour ceux qui n'y participent pas. Et le patient, habitué aux nouvelles victoires, exigera la guérison, pas de rechute; la vérité, pas d'erreur ; les nouveautés les plus en pointe; sinon il courra, éperdu, devant les tribunaux. Il faudrait penser avec assez de profondeur pour éviter de confondre, alors qu'ils s'accompagnent, la rationalisation ou l'objectivité savantes avec ce formatage formidable du social, récemment intervenu.

Ces toutes-puissances imposent silence aux autres valeurs du langage, faibles et méprisées : la supplication amoureuse, celle de pitié, celle de piété. Pouvoirs immanquables, ils ne semblent pas voir que la plupart des hommes, dont je suis, se moquent éperdument du pouvoir, justement, du leur propre comme de tout autre, du performa- 
tif, du séducteur, du Diable et de son train, mais vivent de pitié, d'amour et de piété, toutes relations qui sauvent l'existence et la pensée. Comme tout le monde, j’en vis et j'aurai besoin d'elles et d'elles seulement le moment solennel venu. Je me tournerai alors vers la deuxième tête, celle de pitié.

\section{Bioculture}

Mais avant, je reviens un moment à la guérison. Notre commune francophonie s'exprime de deux manières : ou je guéris de telle maladie ou le docteur m'en guérit. En plaçant donc alternativement le médecin et le malade en position de sujet, cette vibration subtile entre passif et actif, transitif et intransitif dit si bien le mystère de guérir qu'un aphorisme célèbre préféra en laisser prudemment à un Autre l'efficace et la gloire. Je reprends donc l'un des exemples de tantôt : passé les années 1950, les antibiotiques guérirent, et fort activement, certaines maladies infectieuses. Victoire temporaire certes, mais si décisive au regard des impuissances qui la précédaient, que ce verbe guérir et son poids d'histoire et de langue nous empêchent aujourd'hui encore de penser l'événement. Car il faut bien distinguer l'histoire de la médecine et l'anthropologie du corps, dont je viens de parler, d'une autre chose, autrement importante. Que voici.

Depuis que Leeuwenhoek et Swammerdam observèrent l'agitation des spermatozoïdes et autres monocellulaires sous les lentilles des premiers microscopes et que Pasteur, deux siècles plus tard, puis d'autres encore, identifièrent certains microbes comme agents pathogènes, nous confondîmes ennemis et bactéries. La mise à mort des «nuisibles », comme l'on disait dans les livres d'école jadis, et un retour si soudain à la santé nous rendirent aveugles à cette évidence que nous découvrions, en fait, un nouveau règne du vivant, précédant ceux des plantes, animaux, champignons et algues, l'immense monde bactériel, celui auquel des pratiques archaïques avaient vaguement accédé dans le brassage de la bière et les pratiques de la vinification : chantons Noé, par exemple, patriarche digne qui planta la vigne. Au sein de cet univers invisible, grouillant innombrablement et plus vieux que les vivants visibles d'au moins deux milliards d'années, se mêlent " utiles » et «nuisibles », au point que la science rit de ce partage.

Or, et voici le point : quand nous utilisions, pour notre délectation, les ferments ou diastases, ou que nous combattions le bacille de Koch ou le tréponème pâle, nous faisions entrer ce règne sauvage et formidable de nouveaux vivants « naturels » dans la culture. Ce dernier mot, entendez-le alors aussi bien dans le sens noble des philosophes que celui, modeste, des cultivateurs; pour la pensée la plus profonde, seul le second compte.

Car l'agriculture et l'élevage exercent, par rapport aux plantes et aux animaux, autres règnes du vivant, les mêmes opérations que celles auxquelles ce nouveau savoir et cette nouvelle pratique se livrent. Ici comme là, il s'agit, en effet, de favoriser la croissance et le développement de certaines variétés au détriment d'autres. Sauvons le blé des mauvaises herbes, la poule du renard et l'agneau du loup, séparons le domestique en général des espèces chétives, improductives ou sauvages; le chien de berger-pénicilline éloigne ainsi le prédateur ou le parasite pestifere. Bref, nous faisons de même dans le champ de labour, sous le pressoir ou grâce aux médicaments. La sélection artificielle des éleveurs mime celle dite naturelle par Darwin. De Pasteur à Fleming, nous assistons alors à un événement qui dépasse de beaucoup la victoire simplement historique de la santé recouvrée ou l'apparition d'un nouveau corps, pour renouer avec l'origine même de l'agriculture, avant même que commence l'histoire. Et, de nouveau, elle laisse la place à l'évolution. Car cette origine fit bifurquer globalement la condition humaine et celle de tous les vivants, touchés ou non par cet effort sélectif. De même qu'alors les espèces domestiquées de faune et de flore entrèrent en culture, de même, dans les années cinquante, le règne bactériel entra massivement dans l'humain. Nous avons donc vécu le début d'une nouvelle agriculture, celle qui traite de cet invisible proliférant. Quand il s'occupe de la rage et des tartrates, Pasteur doit se lire comme le dernier des savants agraires, pour les chiens et les raisins, mais lorsqu'il découvre les microbes, comme le premier de ces agriculteurs-là. Le laboratoire alors tend à remplacer à la fois vingt thérapies et dix instruments aratoires. Le siècle dernier suscita donc l'avènement d'une bioculture, entendez par ce mot des sciences et pratiques du vivant, tous règnes confondus, visibles et invisibles, algues, champignons, plantes, animaux et bactéries. Jadis, nous dominions vaguement les processus de sélection naturelle; désormais nous dominons, de plus et de façon savante, le hasard des mutations; or la vie additionne sélection plus mutation. Désormais se crée un seul métier, certes indéfiniment spécifié, qui s’occupe de la vie prise en général et dont le naturaliste, le biochimiste, le médecin, le paysan... deviennent des cas particuliers. Dans le futur, que dis-je, aujourd'hui même, la vie dans sa totalité entre dans notre action et notre responsabilité. L'avenir de la médecine se joue à ce niveau évolutif de profondeur. 
Aveuglés par une solution inespérée au problème du mal, nous ne pensions la guérison que dans le temps de l'histoire, alors que se joue un événement de l'ordre au moins de l'hominisation. Adam n'avait nommé que les vivants visibles, l'homme n'avait humanisé que certains de ceuxlà. En généralisant ce geste à la totalité de la vie, nous repassons par l'origine. Cet événement fondateur touche deux parmi les plus vieux métiers du monde, le paysan et le médecin, apparemment dépossédés de leurs pratiques traditionnelles par lesdits formatages, mais surtout par ce choc évolutif. La crise de la médecine se réfere donc à une durée immense et s'associe ainsi à celle de l'agriculture, le plus grand événement du siècle passé. Ce changement, je le nomme ailleurs hominescence.

\section{Retour au portrait : variations sur le thème médecin}

Amis médecins, ce terme qui désigne votre titre me fascine. Car ces deux jumelles : la médication et la méditation vous rendent étonnamment proches des philosophes. En les associant, notre francophonie commune sous-entend qu'il faut penser pour soigner, mais aussi que soigne la pensée, chose moins connue. Cette communauté de mots - qui me donne l'occasion de m'adonner à cet exercice musical connu sous le nom : thème et variations remonte à la haute antiquité indo-européenne, où vous n'exerciez pas, comme ailleurs, le rôle de sorcier, mais celui de sage qui mesure et médite. Saviez-vous que la variation touche aussi au mythe?

L'une des trois Gorgones, la Méduse, la plus lointaine ancêtre connue à porter un nom proche du vôtre, avait le regard fixe de qui force l'attention; combien de fois ai-je admiré la lumière de vos yeux lorsqu'ils scrutaient intensément une part pathétique de mon corps immobilisé. La racine commune de cette famille linguistique, mode, dérive justement de cette mesure, dans son sens raisonnable et modéré, comme une modestie dans le comportement. Vous ne bousculez pas, mais tentez d'accommoder. Pour avoir appris la lenteur des choses et la fragilité des corps, vous n'agissez pas immodérément, ce qui incommoderait. Vos remèdes modifient. A suivre le fil de la langue, vivez-vous comme des modèles?

En variant ainsi sur les mots de la même famille, loin de jouer gratuitement, j'explore le terrain même où se construisent la clinique, l'hôpital ou l'hôtel-Dieu. Car, dans l'ensemble de ces passages entre médecine, remèdes et modes, l'on rencontre les logiques modales, elles-mêmes construites autour de quatre concepts-clés : nécessaire, possible, impossible, contingent. Ils décrivent avec précision le vivant : issue de nécessités physiques, contrainte et réglée par des impossibilités, la vie, conçue dans le possible, se développe et s'épanouit dans la contingence des individus singuliers.

Voilà le résumé de vos tentatives, triomphes et défaites mêlées, votre terminale et sereine mélancolie humaniste : frêle et inventif, fort et faible, victorieux jusqu'à cette heure des obstacles dressés le long de son temps, ce patient pourrait ne pas vivre ; contingent, il essaie mille voies possibles, encadrées durement par force impossibilités, jusqu’à la nécessité irrémédiable de la mort.

Voilà donc les quatre règles de la pratique et de l'éducation médicales, les quatre murs des bâtiments où elle lutte pour la vie. La chimie, la mécanique, les sciences dures encadrent de nécessité une santé, des existences lancées dans la pure possibilité; quel médecin n'a pas vu tous les cas imaginables et même inconcevables? Mais il sait aussi bien qu'expriment dramatiquement nos impossibilités la maladie et la douleur et que l'inéluctable mort nous attend. Pour vous, pour moi, pour nous, le médecin lutte pour le contingent, aux limites du possible et de l'impossible.

\section{L'éducation médicale}

Je reviens, pour finir, à mon début, pour en venir au sujet de notre rencontre. Entre les deux têtes du corps médical, un balancement fit l'histoire. Juste après la seconde guerre mondiale, la première parvint à d'incontestables réussites, dont les résultats firent à mon sens branler même l'évolution et changer l'homme contemporain, en même temps que son environnement physique, vivant et sociétaire. Sur le chemin des victoires, nous avions donc un peu oublié la seconde tête, que je veux, maintenant, magnifier. Face aux bonnes raisons de la science, des statistiques et des politiques de santé publique, face aux formats de l'administration, des médias, de l'argent et de la publicité... le médecin devient le héros -quasi anarchiste !- du singulier, du corps privé, de l'intimité secrète, l'accompagnateur de la souffrance de chacun. Retour de l'archange Raphaël.

Dans le cas contraire, en effet, il ne s'agirait que d'un demi-médecin, qui soignerait les maladies, les classerait, les rangerait, comme des dossiers ; mais l'autre, celui du passé, d'aujourd'hui et du futur, a pansé, soigne et guérira les malades, aime en toutes circonstances les individus sin- 
guliers dont aucune douleur ne ressemble à la voisine ni à celle de sa mère ni à celle de son fils. Le soignant rencontre la singularité comme telle, au moment de sa plus profonde faiblesse, quand la personne même se dévoile, sans mentir, comme elle ne se montra jamais dans l'aise, l'effort ou la joie. Alors que les philosophes ratiocinent indéfiniment sur la définition propre de l'homme, le médecin en contemple le visage et le corps, au voisinage de la mort : ecce homo. Dans la souffrance et l'agonie, ce mot prophétique annonce que l'essence même de cet homme se révèle là. Certes, le praticien en ouïra le cri déchirant au chevet des patients, à l'hôpital ou dans sa clientèle privée. Mais il ne pourra pas toujours le déchiffrer en sa sombre profondeur. Comment apprendre le singulier, sur lequel nul, jamais, ne fit de cours?

Si donc une fée secourable - l'ami Pierre Farah, par exemple - me consultait pour réformer un peu les études médicales, je n'introduirais dans leur cursus qu'un innocent mais indispensable loisir. Comment le praticien futur pourra-t-il soupçonner, en effet, le désespoir d'un amant éconduit s'il n'a pas ouï les Amours de Ronsard; la conduite pathétique d'une veuve douloureuse, que l'entêtement à servir le passé pousse même à des catastrophes aveugles, s'il n'a pas vu Andromaque au théâtre ; les gestes d'un père amoureux de ses filles, jusqu'à la passion auto-destructrice, s'il n'a pas lu le Père Goriot; les risques furtivement pris par une épouse laissée à sa solitude et malade du manque d'amour, s'il ignore Madame Bovary? Un jeune impuissant se raconte dans l'Éducation sentimentale, les épileptiques tombent du haut mal, parmi de merveilleuses auras, dans les romans de Dostoievsky, des mâles détestent les femmes partout, Platon, Abélard et Kant tout autant qu'Adolphe ou Dominique, et veille le paranoïaque dans le labyrinthe sonore et serré du Terrier de Kafka.

Étudiant, mon ami, comment peux-tu apprendre ton art sans cet art, comment équilibrer ta tête à statistiques si tu n'amènes pas l'autre à entrer dans les humanités ? Les grands écrivains travaillèrent pour toi. Depuis l'aurore de la parole, ils traitent, comme toi, du problème du mal, dans le même cadre que toi, la singularité blessée, si pathétique qu'elle oublie même qu'elle va mourir. Sauras-tu comprendre comment bat le cour d'une femme enceinte, si jamais ne batit le tien à ouïr la magnificence du psaume entonné par Marie chez sa cousine Élisabeth lorsque celleci conçut, quoique vieille ? La culture t'apprendra donc la médecine, mieux que l'amphithéâtre, car ces auteurs explorent et décrivent des expériences individuelles telles que tu les rencontreras immanquablement et qu'assurément tu les manqueras si, limité à la raison brute, tu restes un instruit inculte. Le bon médecin tient ses deux têtes pleines et bien faites.

Relis au moins le Livre de Job, les Tragiques grecs et les neuf Béatitudes. 